\title{
Effect of outgassing temperature on the performance of porous materials
}

\author{
Alessandro Figini-Albisetti, Leticia F. Velasco, José B. Parra, Conchi O. Ania* \\ Instituto Nacional del Carbón, CSIC, Oviedo 33080, Spain \\ *Corresponding author E-mail: conchi@incar.csic.es (CO Ania) \\ Tel.: +34985118846; Fax: +34-985297662
}

\begin{abstract}
This work illustrates the consequences of an inadequate outgassing temperature of porous materials of different nature (zeolites and activated carbons) on their performance on gas storage and wastewater remediation. Outgassing at low temperature in thermally stable materials leads to an incomplete cleaning of the porous surface; as a result, the gas storage ability based on adsorption isotherms is underestimated. In contrast, outgassing at elevated temperature in temperature-sensitive materials -such as chemically modified carbons- provokes irreversible changes in the composition (nature and amount of surface groups) and structure of the adsorbents which also affects strongly their stability and performance. Two examples illustrating wrong interpretation data on $\mathrm{CO}_{2}$ capture on zeolites and wastewater treatment using activated carbons are addressed. The results show how the performance of a given material can be significantly modified or misunderstood after outgassing pretreatment.
\end{abstract}

\section{INTRODUCTION}

Porous materials play an outstanding scientific and technological role because of the ability of the pore walls to interact with atoms, ions and molecules, as well as of their capacity to retain liquid and gas molecules in the confined space inside the pores. Driven by the need for highly featured materials in many multidisciplinary areas, recent advances on synthetic routes have enabled to develop materials with unforeseen control over structure and properties that offer unexpected opportunities [1-3] for porous materials. Indeed, in the last decade most research on the design and processing of novel porous materials has aimed to control the size, shape and uniformity of the porous void, contributing to developments in areas beyond the traditional use of porous materials as catalysts and adsorbents.

Besides porosity, surface chemistry of materials provides an unlimited, imaginative and simple tool to face newly arisen environmental challenges. Even small amounts of heteroatoms can determine the physicochemical properties of the materials, and hence, control the desired properties by an adequate functionalization of the material. For instance, the surface functional 
groups determine the self-organization, the chemical stability and the reactivity in adsorptive and catalytic processes [4-5]. Practically any combination of mechanical, electrical, or chemical properties can be achieved by using an adequate precursor and controlling the structure and surface chemistry (i.e., functionalization).

On the other hand, gas adsorption is one of the most widely used techniques for the characterization of porous solids, and exploring gas separation processes and storage capacities. Particularly driven by the increased commercialization of automatic apparatuses, physisorption of gases has become almost a routine technique for the characterization of the porosity. Unfortunately, only few researchers pay attention to the operating conditions for the determination of gas adsorption data, which details are very often omitted in the scientific publications, despite their importance.

This is the case of the outgassing conditions previous to gas adsorption measurements that may lead to a partial decomposition of surface groups having different thermal stability, or to structural decompositions (annealing effects). 'Unconscious' modifications on the material's composition when these are submitted to apparently harmless thermal treatments have more important implications than many users are aware of; and porous materials with high surface heterogeneity and low mechanical resistance should be handled with care when temperature treatments are applied.

The objective of the present work is to emphasize the importance and implications of an adequate choice of operating conditions during the characterization of the porosity of adsorbents, by means of physisorption of gases, particularly on chemically modified materials (i.e., temperature sensitive). Outgassing at elevated temperatures coupled to vacuum may result in irreversible changes in the composition (nature and amount of surface groups) and structure of the activated carbons with a large content of oxygen functionalities. Our purpose is to illustrate the consequences of this effect in the final application of the materials, concerning $\mathrm{CO}_{2}$ storage capacity and wastewater remediation, showing how the performance of a given material can be significantly modified or misunderstood after outgassing pretreatment.

\section{MATERIALS AND METHODS}

\subsection{ADSORBENTS}

We have selected two highly porous adsorbents for our study: LTA-4A zeolite (labeled as LTA4A) and an activated carbon (P) prepared from physical activation under $\mathrm{CO}_{2}$ atmosphere (33 \% burn-off degree) of a plastic waste. Details on the synthesis procedure have been reported in earlier works [6]. One of the characteristics of this material is that it is mainly composed of carbon (ca. > 99.5 wt. \% C) due to the absence of mineral matter and other impurities in the composition of the precursor, thus it can be considered as a model carbon material. In order to 
increase the surface heterogeneity, $\mathrm{P}$ carbon was oxidized with $\left(\mathrm{NH}_{4}\right)_{2} \mathrm{~S}_{2} \mathrm{O}_{8}$ (sample PS) following the procedure described elsewhere [7]. The oxidized sample was washed in hot distilled water in a Soxhlet apparatus and dried at $60^{\circ} \mathrm{C}$ overnight.

\subsection{CHARACTERIZATION}

The adsorbents were submitted to textural and chemical characterization by means of physisorption of $\mathrm{N}_{2}$ at $-196{ }^{\circ} \mathrm{C}$ and $\mathrm{CO}_{2}$ at $0{ }^{\circ} \mathrm{C}$ (Micromeritics 2010 and Tristar 3000). Gas adsorption isotherms were performed in duplicate, being each run measured on a fresh aliquot of material. Previously, the samples were outgassed under high vacuum (ca. $10^{-7}$ torr) during 17 hours and at different temperatures $\left(25,120,250\right.$ and $\left.400{ }^{\circ} \mathrm{C}\right)$. The samples were labeled as the initial of the adsorbent (LTA4A, P or PS) followed by "outX" where out stands for outgassed and $\mathrm{X}$ for the temperature of outgassing. For instance, PSout25 refers to the oxidized carbon after outgassing under vacuum at $25{ }^{\circ} \mathrm{C}$. Another aliquot of the oxidized carbon was heated at $800^{\circ} \mathrm{C}$ under nitrogen atmosphere $\left(5^{\circ} \mathrm{C} \mathrm{min}{ }^{-1}, 1 \mathrm{~h}\right)$ to remove most of the surface functionalities. This sample was labeled as PSH800. The effect of the thermal treatment on the density of surface groups of the carbons was also followed by thermal analysis in a Setaram

Labsys instrument. Temperature programmed desorption (TPD) experiments were carried out under a nitrogen flow rate of $50 \mathrm{~cm}^{3} \mathrm{~min}^{-1}$, at a heating rate of $15{ }^{\circ} \mathrm{C} \mathrm{min}^{-1}$, up to a final temperature of $900^{\circ} \mathrm{C}$. For each experiment, about $30 \mathrm{mg}$ of carbon sample was used.

\subsection{ADSORPTION FROM SOLUTION}

Details of the experimental procedure for evaluating the adsorption capacities from solution have been reported elsewhere [8]. Briefly, phenol uptake was evaluated from dynamic adsorption tests obtained at $25^{\circ} \mathrm{C}$ using ca. $300 \mathrm{mg}$ of carbon sample in a $5 \mathrm{~cm}$ length stainless steel column at a flow rate of $4 \mathrm{~mL} \mathrm{~min}^{-1}$. The initial concentration of phenol was $1000 \mathrm{mg} \mathrm{L}^{-1}$, and the corresponding adsorption capacities were obtained from integration of the breakthrough curves at the saturation point. The adsorption tests were carried out in the initial carbons ( $\mathrm{P}$ and PS) before and after the outgassing treatments at various temperatures.

\section{RESULTS AND DISCUSSION}

As mentioned above, gas adsorption has become a popular technique for many applications such as the characterization of porous solids, and gas storage and separation processes of industrial interest. However, little attention is currently being paid to the experimental procedures for the determination of gas adsorption measurements, and the implications on the performance of the investigated materials.

Quoting IUPAC recommendations for reporting gas physisorption data on solid/gas systems [9, 10], 'prior to the determination of an adsorption isotherm most if not all of the physisorbed 
species must be removed from the surface of the adsorbent. This may be achieved by outgassing, i.e. exposure of the surface to a high vacuum - usually at elevated temperature'. Nevertheless, very often the experimental details on the determination of gas adsorption isotherms are omitted, and even neglected. What is seriously troubling is that this leads to crucial experimental errors and hence the conclusions derived from such neither rigorous nor reliable studies are erroneous. For illustrating the importance of the outgassing temperature on the performance of microporous materials, we have investigated a zeolite and an activated carbon applied for gas storage and wastewater remediation, respectively.

Zeolite LTA4A was used to evaluate its $\mathrm{CO}_{2}$ adsorption capacity. The adsorption isotherms were determined by outgassing the zeolite at 25,120 and $350{ }^{\circ} \mathrm{C}$ (Fig. 1). Although in all cases the isotherms were perfectly reproducible, there are clear differences between them. As the outgassing temperature is raised, the amount of $\mathrm{CO}_{2}$ adsorbed increased significantly. The lowest adsorption capacity was achieved on the sample outgassed at $25^{\circ} \mathrm{C}$, which is due to an incomplete evacuation of the moisture retained on the zeolite network. This is in good agreement with the thermal analysis of the as-received (non dried) zeolite (Fig. 2A) where the peak due to the water physisorbed is centered around $200^{\circ} \mathrm{C}$. Being thermally stable, LTA4A zeolite should be outgassed at temperatures above $200{ }^{\circ} \mathrm{C}$ (ideally $300-400{ }^{\circ} \mathrm{C}$ ) to clean the surface from any adsorbed impurity.

Comparing our data with that reported by other research groups [11-13] in the literature, it is demonstrated that a complete evacuation of the zeolite is not achieved unless high temperature coupled to high vacuum is applied. An important implication derived from this fact is that the $\mathrm{CO}_{2}$ adsorption capacity obtained from the isotherm measured at $25^{\circ} \mathrm{C}$ is underestimated. In a previous work, we have used this experimental data for the validation of a force-field for predicting the adsorption capacity in zeolites [14], obtaining very successful results when compared to those reported in the literature $[12,13]$. The success of our force-field relied on the quality of the experimental data used for validation (reliability, accuracy and reproducibility), thus affording for the first time a fully transferable force-field between different zeolite framework types for the prediction adsorption properties. In contrast, previously reported force-fields had failed to reproduce the adsorption capacities of zeolites with different topologies and compositions as they were fitted on wrong experimental datasets $[12,13]$ due to an incomplete evacuation of the zeolites.

However, outgassing at high temperature cannot be applied to temperature-sensitive materials, susceptible to undergo irreversible changes on their structure. This is the case of porous carbon materials that exhibit a heterogeneous surface chemistry. To illustrate the effects of outgassing temperature on activated carbons we have investigated two activated carbons -P and PS-, characterized by different acidic/basic nature. Both as-received and outgassed samples were 
used for the adsorption of phenol from aqueous solution, in order to investigate the effects of the outgassing on the performance towards the uptake of pollutants from liquid phase.

For the most hydrophobic carbon (sample P), we observed that the outgassing conditions affect the textural characterization of the adsorbent. Increasing the degassing temperature also rises the amount of $\mathrm{N}_{2}$ and $\mathrm{CO}_{2}$ adsorbed (Table 1), indicating that despite its hydrophobic nature, the removal of moisture and other impurities from the carbon surface is not complete unless high temperatures are applied. Consequently, textural data reported for Pout25 and Pout120 is incomplete and not accurate, as it corresponds to a partial cleaning of the surface. In contrast, the isotherms of the samples outgassed at 250 and $400{ }^{\circ} \mathrm{C}$ practically overlap over the whole range of relative pressures. This indicates that $250{ }^{\circ} \mathrm{C}$ should be high enough for an accurate textural characterization of the porosity of this material. Further increase of the temperature (i.e., $800{ }^{\circ} \mathrm{C}$ ) did not change the isotherm for carbon P; however, it should be misadvised for many carbon materials due to potential annealing effects of the structure [15].

Such small differences observed in the textural characterization do not seem to affect the adsorption capacity of $\mathrm{P}$ carbon from solution. Phenol uptake after outgassing hydrophobic $\mathrm{P}$ carbon at the different temperatures $\left(25,120,250\right.$ and $\left.400{ }^{\circ} \mathrm{C}\right)$ did not vary $\left(245 \mathrm{mg} \mathrm{g}^{-1}\right)$, with a relative standard deviation below $5 \%$. Even after heat treatment at $800{ }^{\circ} \mathrm{C}$, the removal capacity remained almost constant. This confirms that for hydrophobic carbons, degassing temperature only affects the adsorption capacity of gases due to occluded gases that remain inside the porosity, but not the retention of aromatic compounds from diluted solutions.

In contrast, the effect on the hydrophilic PS carbon is more remarkable. First of all, degassing at room temperature under vacuum proceeded very slowly, due to the moisture retained by this carbon; even after 17 hours of outgassing, the vacuum gauge remained at relatively high values (above 80 torr). Therefore the degassing treatment needs to be performed at higher temperatures. The question is how far the temperature may go without provoking irreversible changes in the samples.

To evaluate this issue, TG profiles of the samples outgassed at various temperatures have been performed (Fig. 2B) and the mass loss of the corresponding samples evaluated (Table 2). The profiles of the non-outgassed carbons (P and PS) are also included for comparison. The first peak centered at temperatures below $150^{\circ} \mathrm{C}$ that appears in all the profiles has been assigned to the moisture retained on the carbons. To counterbalance this effect of moisture on the mass loss, all calculations in Table 2 are performed from $150^{\circ} \mathrm{C}$; thus any changes in the mass loss can only be attributed to modifications in the carbon due to decomposition of surface groups during outgassing.

As mentioned before, even in the case of the hydrophobic P carbon the moisture physically adsorbed is not completely removed until moderate temperatures are applied. For the hydrophilic carbon the changes occurred during degassing are more evident, even at room 
temperature. It appears that not only the adsorbed moisture is removed during the outgassing at room temperature; the intensity of the low temperature peaks in the TG profile fell by 2 times as compared to that in PS, suggesting the removal of a small amount of surface groups. When the temperature increases, the composition of the sample suffers more notable changes. For instance, the total mass loss decreased from $20 \%$ in the non-degassed PS sample down to $9.6 \%$ in the case of PSout400 (Table 2); this fact can only be attributed to the partial decomposition of the oxygen functionalities. A similar behavior has been observed in other temperature sensitive materials although due to different reasons. In the case of amorphous and poorly crystalline minerals, outgassing at elevated temperatures prior to BET measurements provokes phase changes [16].

The extent of the decomposition of the functionalities strongly depends on the temperature applied, and affects not only the amount but also the nature of the surface groups. Labile groups decompose at temperatures below $400{ }^{\circ} \mathrm{C}$, whereas an almost complete withdrawn of the functionalities occurred after the treatment at $800^{\circ} \mathrm{C}$ [17].

Regarding the textural characterization, the porous features of the studied carbons vary with the outgassing temperature (Table 1). The nitrogen adsorption isotherms of the oxidized PS carbon submitted to the different outgassing treatments are parallel, although downward shifted. The largest swing is obtained for PSout25, and gradually becomes smaller as the temperature is raised. This affects the textural parameters obtained from the isotherms; for instance, a decrease by $27 \%$ in the surface area and pore volumes was observed for carbon PSout120 compared to PSout25. The subsequent partial outgassing increased the surface area, although the values are not completely restored compared to those of the non-oxidized $\mathrm{P}$ carbon. The same trend was obtained for the values of total pore volume and micropore volume. A similar observation on the increase in the textural parameters after heat treatment of oxidized carbons has been reported in the literature [18]. These results demonstrate that the porosity measured by gas adsorption in oxidized carbons is linked to the outgassing temperature.

At the same time, it should not be forgotten that the nature and amount of surface groups undergo important changes upon the degassing pretreatment (Table 2 and Fig. 2B). Therefore, one should take into account before the consequences in the likely modification in the composition of the adsorbent, before its utilization for a given application. In this regard, a recent paper by Carrott and co-workers has reported the significant effect of degassing temperature on the performance of carbon molecular sieves for gas separation [19]. Those results have shown that the selectivity of the separation of various gases can be somehow tuned depending on the degassing pre-treatment. In the case of liquid phase applications, this issue may become more critical, as the as-received materials used for the removal from liquid phase are not those that have been outgassed and which textural characterization is being reported. 
To illustrate this point, we have performed dynamic adsorption tests for evaluating the phenol uptake of the outgassed carbons from aqueous solution (Fig. 3). The values of the corresponding adsorption capacities evaluated by integration of the breakthrough curves are also indicated in Fig. 3 in boxes. Phenol adsorption capacity strongly depends on the nature of the carbons, the highest uptake being reached on the most hydrophobic carbon (sample P). Oxidation of the carbon dramatically decreased phenol retention, which is in good agreement with well-known earlier works on the removal of phenolic compounds [4, 7]. What is importantly shown in this paper is that the outgassing temperature chosen for the routine textural characterization of the adsorbents, causes irreversible changes in the chemical nature and porosity of temperature-sensitive materials and thus the performance of the carbon is also modified.

For instance, outgassing hydrophobic $\mathrm{P}$ carbon at 25,120 or $250^{\circ} \mathrm{C}$ did not affect phenol removal capacity ( $245 \mathrm{mg} \mathrm{g}^{-1}$ ), with a relative standard deviation below $5 \%$. On the other hand, a different trend is obtained for the hydrophilic carbon. Outgassing at room temperature (PSout25) gives rise to an increase by almost $75 \%$ in the adsorption capacity if compared to the parent oxidized PS carbon. This result gives an idea of the drastic effect of degassing on the retention capacity, therefore on the adequate choice of the parameters used in routine characterization techniques when temperature sensitive carbons are handled. A typical outgassing temperature applied for measuring the porosity of carbon adsorbents (i.e., $250{ }^{\circ} \mathrm{C}$ ) brings about an increase in the adsorption capacity by almost 3 times, if compared to carbon PS.

\section{CONCLUSIONS}

This work illustrates the consequences of an inadequate outgassing temperature of porous materials on their performance on gas storage and wastewater remediation. Outgassing at low temperature in thermally stable materials leads to an incomplete cleaning of the porous surface; as a result, the gas storage ability based on adsorption isotherms is underestimated. In contrast, outgassing at elevated temperature in temperature-sensitive materials provokes irreversible changes in the composition and structure of the adsorbents which may affect their stability and performance. Consequently, it is necessary to conduct a brief analysis of the surface groups (nature and stability) of a given porous material before choosing an adequate outgassing temperature during routine gas adsorption measurements. Outgassing temperature has to be also consistent with the final application of the material. Otherwise, the obtained data may be reproducible but neither accurate nor reliable.

\section{ACKNOWLEDGMENTS}

This work was supported by the Spanish MICINN (CTM2008-01956). COA thanks her Ramon y Cajal Research Contract. LFV thanks her JAE predoctoral fellowship. 


\section{REFERENCES}

[1] I. Kiricsi, J.B. Nagy, H.G. Karge, Gyula Palyi, (Eds.) in Porous materials in environmentally friendly processes, Elsevier, Amsterdam, 2002.

[2] M.L. Occelli in Synthesis of Porous Materials: Zeolites, Clays and Nanostructures (Chemical Industries), Marcel and Dekker Inc., New York, 1996

[3] Y. Gogotsi (Ed.) in Carbon Nanomaterials, CRC Press, Taylor \& Francis Group, 2006, Boca Raton, Florida.

[4] T.J. Bandosz (Ed.) in Activated carbon surfaces for environmental remediation, in the series Interface Science and Technology, Elsevier, 2006, New York.

[5] J.A. Schwartz, C. Contescu (Edts.) in Surfaces of Nanoparticles and Porous Materials (Surfactant Science) Marcel \& Dekker, New York, 1999.

[6] J.B. Parra, C.O. Ania, A. Arenillas, F. Rubiera, J.J. Pis, High value carbon materials from PET recycling, Appl. Surf. Sci., 238 (2004) 304.

[7] C.O. Ania, J.B. Parra, J.J. Pis, Oxygen-induced decrease in the equilibrium adsorptive capacities of activated carbons, Ads. Sci. Technol., 22 (2004) 337.

[8] C.O. Ania, J.B. Parra, J.A. Menendez, J.J. Pis, Microwave-assisted regeneration of activated carbons loaded with pharmaceuticals, Wat. Res., 41 (2007) 3299.

[9] J. Rouquerol, D. Avnir, C.W. Fairbridge, D.H. Everett; J.H. Haynes, N. Pernicone, J.D.F. Ramsay, K.S.W. Sing, K.K. Unger, Recommendations for the characterization of porous solids (Technical Report), Pure Appl. Chem., 66 (1994) 1739-1758.

[10] K.S.W. Sing, Reporting physisorption data for gas/solid systems with Special Reference to the Determination of Surface Area and Porosity, Pure Appl. Chem., 54 (1982) 2201.

[11] H. Ahn, J.H. Moon, S.H. Hyun, C.H. Lee, Diffusion Mechanism of Carbon Dioxide in Zeolite 4A and CaX Pellets, Adsorption 10 (2004) 111-128.

[12] E.D. Akten, R. Siriwardane, D.S. Sholl, Monte Carlo Simulation of Single- and BinaryComponent Adsorption of $\mathrm{CO}_{2}, \mathrm{~N}_{2}$, and $\mathrm{H}_{2}$ in Zeolite Na-4A, Energy Fuels 17 (2003) 977-983.

[13] E. Jaramillo, M. Chandross, Adsorption of small molecules in LTA zeolites. 1. $\mathrm{NH}_{3}, \mathrm{CO}_{2}$, and $\mathrm{H}_{2} \mathrm{O}$ in zeolite 4A, J. Phys. Chem. B 108 (2004) 20155-59.

[14] A. Garcia-Sanchez, C.O. Ania, J.B. Parra, D. Dubbeldam, T.J.H. Vlugt, R. Krishna, S. Calero, Transferable Force Field for Carbon Dioxide Adsorption in Zeolites, J. Phys. Chem. C 113 (2009) 8814-8820.

[15] C.O. Ania, J.B. Parra, J.A. Menendez, J.J. Pis, Effect of microwave and conventional regeneration on the microporous and mesoporous network and on the adsorptive capacity of activated carbons, Micropo. Mesop. Mater. 85 (2005) 7-15

[16] L. Clausen, I. Fabricius, BET Measurements, outgassing of minerals, J. Colloid Interf. Sci. 227 (2000) 7.

[17] S. Haydar, C. Moreno-Castilla, M.A. Ferro-García, F. Carrasco-Marín, J. Rivera-Utrilla, A. Perrard, J.P. Joly, Regularities in the temperature-programmed desorption spectra of $\mathrm{CO}_{2}$ and $\mathrm{CO}$ from activated carbons, Carbon 38 (2000) 1297.

[18] S. Haydar, M.A. Ferro-García, J. Rivera-Utrilla, J.P. Joly, Adsorption of p-nitrophenol on an activated carbon with different oxidations, Carbon 41 (2003) 387

[19] I.P.P. Cansado, M. Ribeiro Carrot, P.J.M. Carrott, Influence of Degassing Temperature on the Performance of Carbon Molecular Sieves for Separations Involving $\mathrm{O}_{2}, \mathrm{~N}_{2}, \mathrm{CO}_{2}$ and $\mathrm{CH}_{4}$, Energy \& Fuels 20 (2006) 766-770. 


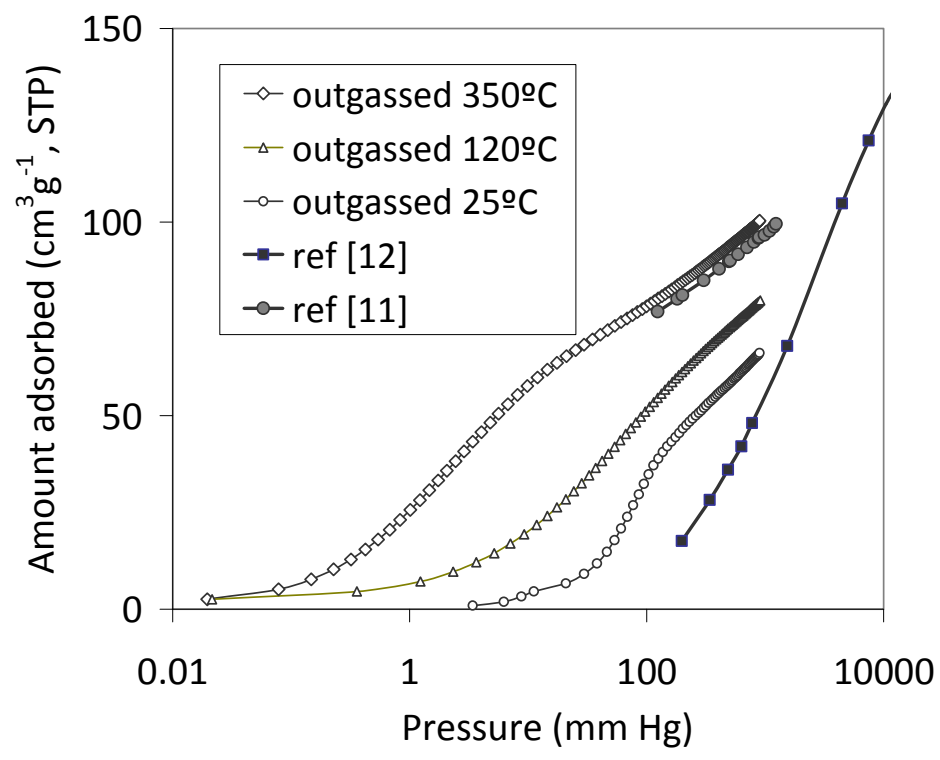

Figure 1. $\mathrm{CO}_{2}$ adsorption isotherms at $25{ }^{\circ} \mathrm{C}$ of zeolite LTA4A after degassing at various temperatures and high vacuum. Experimental data available in the literature obtained at different conditions $[11,12]$ is also plotted for comparison purposes. 

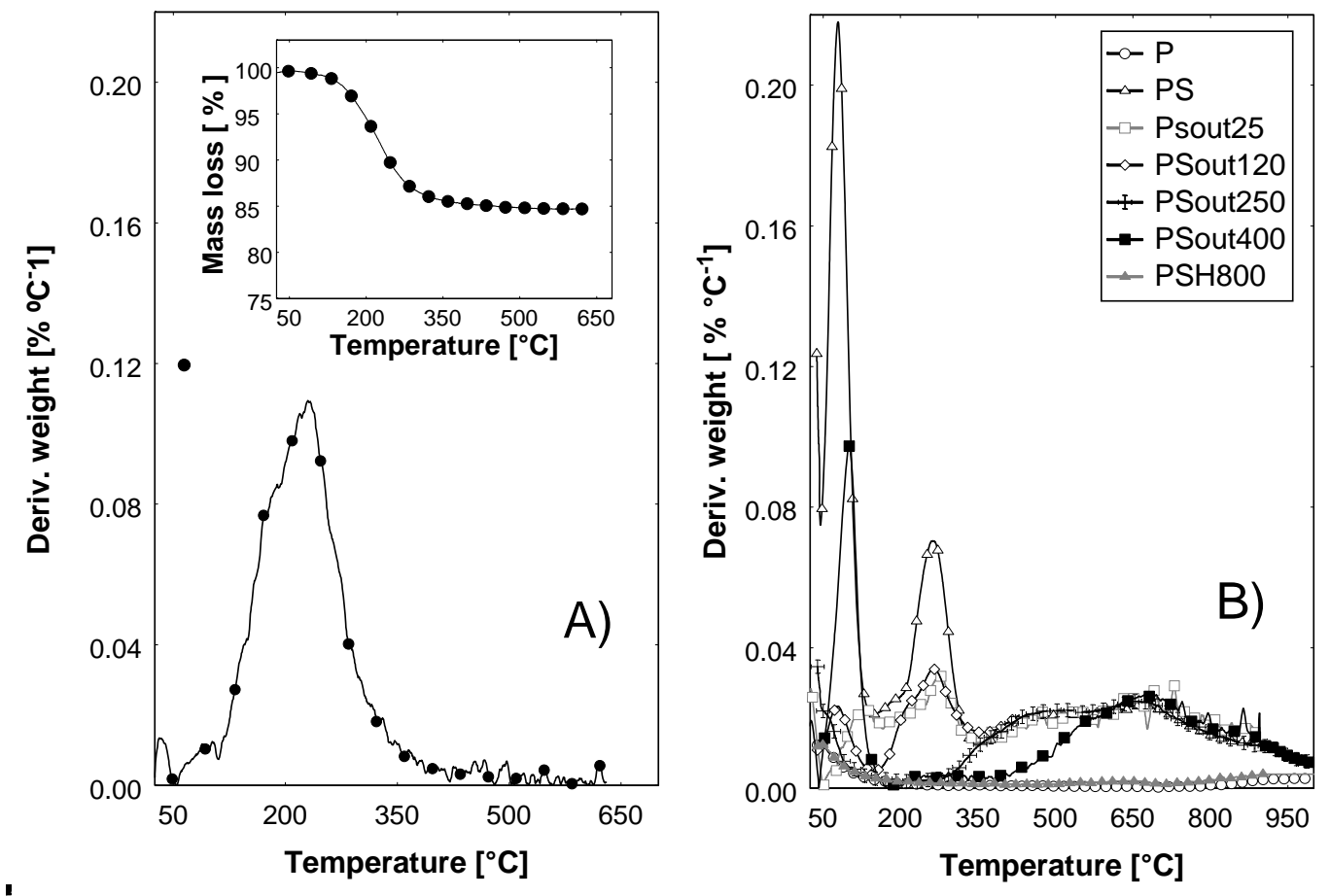

Figure 2. Thermogravimetric profiles of the samples submitted to different outgassing temperatures. A) LTA4 zeolite and B) parent and oxidized carbons.

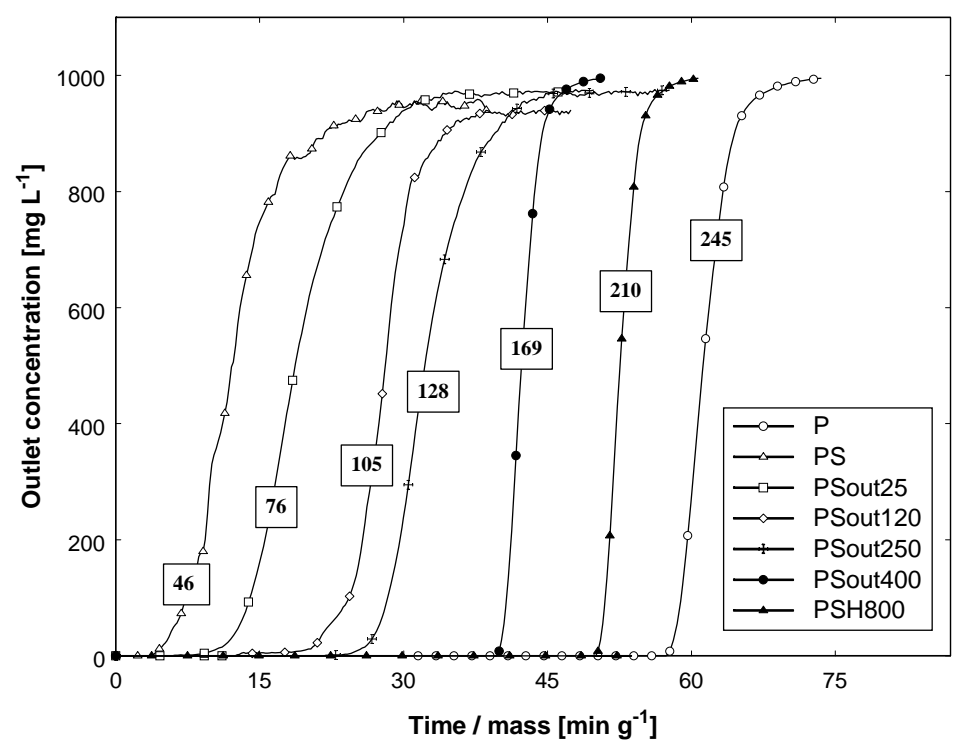

Figure 3. Phenol saturation breakthrough curves on the studied samples. Mass of adsorbent ca. $400 \mathrm{mg}$, initial solution concentration $1000 \mathrm{mg} \mathrm{L}^{-1}$, flow rate $4 \mathrm{~mL} \mathrm{~min}^{-1}$. Boxes indicate phenol uptake in terms of mg adsorbed per gram of adsorbent [ $\left.\mathrm{mg} \mathrm{g}^{-1}\right]$. 
Table 1. Main textural parameters of the samples outgassed at different temperatures, obtained from nitrogen and $\mathrm{CO}_{2}$ adsorption isotherms at -196 and $0^{\circ} \mathrm{C}$, respectively

\begin{tabular}{lccccc}
\hline \multicolumn{1}{c}{ Sample } & $\begin{array}{c}\mathbf{S}_{\text {BET }} \\
{\left[\mathbf{m}^{\mathbf{2}} \mathbf{g}^{-\mathbf{1}} \mathbf{]}\right.}\end{array}$ & $\begin{array}{c}\mathbf{V}_{\text {TOTAL }} \\
{\left[\mathbf{c m}^{\mathbf{3}} \mathbf{g}^{-\mathbf{1}} \mathbf{]}^{\mathbf{A}}\right.}\end{array}$ & $\begin{array}{c}\mathbf{V}_{\text {MICROPORES }} \\
{\left[\mathbf{c m}^{\mathbf{3}} \mathbf{g}^{-\mathbf{1}}\right]^{\mathbf{B}}}\end{array}$ & $\begin{array}{c}\mathbf{V}_{\text {MESOPORES }} \\
{\left[\mathbf{c m}^{\mathbf{3}} \mathbf{g}^{-\mathbf{1}} \mathbf{]}^{\mathbf{B}}\right.}\end{array}$ & $\begin{array}{c}\mathbf{W}_{\text {CO2 }} \\
{\left[\mathbf{c m}^{\mathbf{3}} \mathbf{g}^{-\mathbf{1}}\right]^{\mathbf{C}}}\end{array}$ \\
\hline Pout25 & 1044 & 0.358 & 0.331 & 0.031 & 0.285 \\
Pout120 & 1069 & 0.463 & 0.350 & 0.033 & 0.307 \\
Pout250 & 1235 & 0.546 & 0.411 & 0.032 & 0.341 \\
Pout400 & 1238 & 0.546 & 0.413 & 0.033 & 0.338 \\
\hline PSout25 & 696 & 0.285 & 0.228 & 0.006 & 0.275 \\
PSout120 & 782 & 0.327 & 0.256 & 0.016 & 0.296 \\
PSout250 & 851 & 0.361 & 0.280 & 0.021 & 0.297 \\
PSout400 & 893 & 0.381 & 0.291 & 0.022 & 0.273 \\
PSH800 & 943 & 0.404 & 0.313 & 0.023 & 0.260 \\
\hline
\end{tabular}

${ }^{\text {A }}$ Total pore volume evaluated at $\mathrm{p} / \mathrm{p}^{0} \sim 0.99$

${ }^{\mathrm{B}}$ evaluated from DFT method applied to $\mathrm{N}_{2}$ adsorption data at $-196^{\circ} \mathrm{C}$

${ }^{\mathrm{C}}$ Evaluated from DR equation applied to $\mathrm{CO}_{2}$ adsorption data at $0^{\circ} \mathrm{C}$

Table 2. Mass loss evaluated from the TG profiles of the studied activated carbons

\begin{tabular}{lccc}
\hline & {$\left[\mathbf{1 5 0 - 9 0 0}^{\circ} \mathbf{C}\right]$} & {$\left[\mathbf{1 5 0 -}^{-350}{ }^{\circ} \mathbf{C}\right]$} & {$\left[\mathbf{3 5 0 - 9 0 0}^{\circ} \mathbf{C}\right]$} \\
\hline P & 0.9 & 0.7 & 0.2 \\
PS & 20.5 & 8.1 & 12.4 \\
\hline PSout25 & 16.8 & 4.5 & 11.7 \\
PSout120 & 16.4 & 4.2 & 12.2 \\
PSout250 & 12.1 & 0.8 & 11.3 \\
PSout400 & 9.6 & 0.5 & 9.1 \\
PSH800 & 1.4 & 0.4 & 1.0 \\
\hline
\end{tabular}

